\title{
STUDY OF CROSSOVER OPERATORS OF GENETIC ALGORITHM\& DEVELOPMENT OF NEW CROSSOVER OPERATOR TO SOLVE HETEROGENEOUS VEHICLE ROUTING PROBLEM WITH TIME WINDOWS
}

\author{
Sachin Kumar Nagle and Panneerselvam R \\ Department of management Studies, \\ School of Management, Pondicherry University, Pondicherry, India.
}

\begin{abstract}
Heterogeneous Vehicle Routing Problem with Time Windows (HVRPTW) is amore practical variant of Classical Vehicle Routing Problem. The complexity of solving this problem increases exponentially as it is defined as N-P hard combinatorial problem. Genetic algorithm is widely applied Nobel algorithm and it is a very effective to solve such kind of problems. Genetic algorithm generates near optimum results with less computational time. The effectiveness of genetic algorithm relies on its operator's such as selection, crossover and mutations. The main objective of this paper is to study various crossover operators of genetic algorithm and develop a new crossover operator to solve HVRPTW.
\end{abstract}

Key words: Genetic Algorithm, Crossover Operators, Heterogeneous Vehicle Routing Problem and Time Windows.

Cite this Article: Sachin Kumar Nagle and Panneerselvam R, Study of Crossover operators of Genetic Algorithm\& Development of New Crossover Operator to Solve Heterogeneous Vehicle Routing Problem with Time Windows, International Journal of Production Technology and Management (IJPTM), 9(2), 2018, pp. 1-22. http://iaeme.com/Home/issue/IJPTM?Volume=9\&Issue=2

\section{INTRODUCTION}

The growth of an organization depends on activities, via. Supply Chain Management (SCM) involving the movement and storage of resources from the point of origin to the point of consumption. Due to its importance, many companies are trying to develop efficient methods of SCM to increase customer satisfaction and reduce cost. One of the most important problems associated with SCM is the Vehicle Routing Problem (VRP).The VRP was introduced by Dantzig and Ramser (1959) aiming to determine an optimal route for homogeneous vehicles to serve a set of customers such that each vehicle's route starts and ends at the same depot and 
each customer is served exactly once by one vehicle. One of the most practical extensions of VRP is the Heterogeneous Vehicle Routing Problem (HVRP).

HVRP was introduced by Golden et al. (1984) and it has evolved as a crucial field of research. It is a combinatorial problem and extension of VRP that aims to serve a group of customers with minimum cost and a limited number of vehicles having different capacities. Other extensions of HVRP include studies like Dondo et al. (2007), Xu et al. (2012) and Afsharnadjafi et al. (2014), who considered the multi-depot routing problem with time windows and heterogeneous vehicles. De et al. (2013), Amador-Fontalvo et al. (2014) and Kabcome et al. (2015) present soft time windows and distribution of products with multiple categories. In this context, Jabali et al. (2016) and Nagle and Pannerselvam (2016) provide an extensive survey of literature on HVRP and its variants.

The HVRPTW is a more practical approach of SCM in the context of present scenario with the increased flow of goods to satisfy customer demand. The ultimate objective of this problem is to determine the optimal route of heterogeneous vehicles in order to minimize the total operating cost of the vehicles, while satisfying all the constraints of Clas sical Vehicle Routing Problem (CVRP) and other constraints like, service of each customer must start within the customer time window, each route starts at a depot and ends at the same depot. The total demand of customers served in each route must not exceed the capacity of the associated vehicle. There are some mathematical models in literature to solve it. Since, the models are not appropriate to solve big size combinatorial problems, meta-heuristics like Genetic Algorithm (GA) with new reproduction strategies is highly necessary for this case. GA is very effective in solving such kind of problems but the effectiveness of GA depends on its operators such as selection, crossover and mutations. So the objective of this paper is to study about the crossover operators of GA and develop a new crossover operator to solve HVRPTW.

\section{LITERATURE REVIEW}

Puljic and Manger (2013) presented a comparison of eight evolutionary operators for the vehicle routing problem. These operators are order crossover (OX), partially mapped crossover (PMX), edge recombination crossover (ERX), alternative edges crossover (AEX), heuristic greedy or random or probabilistic crossover (HGrsX or HRndX or HProX), mutation by inversion (IM), mutation by reinsertion (RM) and swap mutation (SM). These crossover operators are tested on a set of well-known benchmark problem instances. According to their comparison, all the above operators are appropriate for the VRP and they yield better results when they are joined together, but if a single operator is used, then a better performance is provided by HGreX or AEX. Appropriate results are obtained by HProX. On the other hand, PMX and CX provide remarkably poor solutions. Haj-Rachid et. al. (2010) studied the performance of crossover and mutation operators for VRP. In their study, they compared four crossover operators, viz. PMX, merge 2 crossover (MX2), OX, and Uniform crossover (UX). They also used three mutation operators with similar mutation rate, namely inversion mutation (1\%), swapping mutation (1\%) and Swap-inverse (swap (1\%) and inverse (1\%)) together. After comparison, they provided an order from best to worst for CVRP, which is PMX, OX, UX and MX2. Ombuki et. al. (2006) developed a problem based specific Best Cost Route Crossover method (BCRC), which minimises the number of vehicles and cost simultaneously, while satisfying all the constraints. They solved Multi-Objective GA (MOGA) for the vehicle routing problem with time windows by using the above crossover operator and got remarkable results. Pierre and Zakaria (2014) introduced partially optimized cyclic shift crossover (POCSX) for (MOGA) for the multiobjective vehicle routing problem with time windows. They compared MOGA based on POCSX with MOGA (BCRC). The comparison shows that the result generated by MOGA based on POCSX is better than the result generated by MOGA based on 
BCRC. Pierre and Zakaria (2017) presented a stochastic partially optimized cyclic shift crossover operator (SPOCSX). This is an enhancement of the partially optimized cyclic shift crossover (POCSX), which is initially presented and combined into MOGA for the optimization of the multi-objective vehicle routing problem with time windows using genetic algorithm. They introduced SPOCSX to overcome the weaknesses of POCSX. The analysis based on the benchmark problems shows that the new features do not affect the computational time. They concluded that the solutions provided by the SPOCSX are much better than the solutions provided POCSX. Also, the analysis shows that the quality of the solutions obtained by SPOCSX are inexpensive than the solutions obtained by its insertion-based counterpart, the BCRC. Berger and Barkaoui (2004) considered the insertion based crossover operator (IB_X) and insert in route neighbourhood (IRN_X) for a parallel hybrid GA for the vehicle routing problem with time windows. Computational results show that the parallel hybrid GA based on IB_X and IRN_Xgenerates cost-effective and very competitive results. Blanton Jr. and Wainwright (1993) presented two new crossover operators, viz. Merge cross 1 (MX1) and Merge cross 2 (MX2). They compared these operators with PMX, CX and ERC, and concluded that MX2 operators are excellent for solving the problem, where there exists a global precedence relationship of the genes. Chand and Mohanty (2013) introduced sub route sequence crossover method and sub route alter mutation method. They solved the real-time vehicle routing problem with time windows and simultaneous delivery of products and pickup of wastage materials. The comparative result shows that the proposed method yields better result when it is compared with classical algorithm. Anand and Panneerselvam (2016) studied different crossover operators and proposed a new crossover operator called as ThreeChromosome Juggling Crossover (TCJC) Operator for genetic algorithm to solve open shop scheduling problem in which the objective is to minimize the makepsan. Varunkumar and Panneerselvam (2017) studied different crossover operators and proposed a new crossover operator called as Sinusoidal Motion Crossover Operator for genetic algorithm to solve vehicle routing problem and its variants.

Some others scholars also solved different kinds of problem by developing different crossover operators for the genetic algorithm. Vijaymurthi and Panneerselvam (2010) developed a GA based Heuristic for total covering problem (TCP), which is a facility location problem in which a minimum number of facilities is to be located to serve a given set of customers subject to satisfying a maximum distance criterion. If a customer is located within that distance with reference to the location of a facility, then that customer will be served by that facility. They developed a Care and Share crossover method, which is new. They compared the results of this algorithm with those of an algorithm developed by Panneerselvam (1996) using bench mark data and found that the new algorithm performs better than the earlier algorithm. Senthilkumar and Narayanan (2011) developed a Genetic Algorithm (GA) based heuristic to schedule the jobs of uniform parallel machine scheduling problem with the objective of minimizing the makes pan. They used single point crossover method and two point crossover method in the skeleton of the standard genetic algorithm to device four different new genetic algorithms and compared them using a complete factorial experiment with three factors, viz. Problem Size, Crossover Method and Job Allocation Method. They found that the GA based heuristic with single point crossover method, in which the proportionate number of jobs allocated to machines based on machine speeds, performs better than the other algorithms. Sivasankaran and Shahabudeen (2014) developed a set of GA-Based heuristics applied to assembly line balancing problem. The main objective of this problem is to assemble the tasks in the assembly system into a minimum number of workstations for a given cycle time in order to maximise the balancing efficiency. For solving this problem, they considered three crossover methods, viz. cyclic crossover method, Forward crossover method, Reverse crossover method. They compared the results of their proposed algorithms through a complete factorial experiment 
with three-factor, viz. Problem size, Cycle time and Algorithm. They found that these factors have the effect on the balancing efficiency. Hence, through Duncan's multiple range test, they found that GA-based heuristic with the forward crossover method is proved to be superior to all other algorithms proposed in their research.

Aravendan and Panneerselvam (2015) developed hybrid genetic algorithms for network design problem in closed-loop supply chain. They proposed a set of genetic algorithms by treating crossover method and selection method as variants. They compared these algorithms and identified the best genetic algorithm among them. Further, they compared the best genetic algorithm with the results of a mathematical model using small and moderate size problems and found that there are no significant differences among the best genetic algorithm and the mathematical model in terms of total cost for the design of supply chain network. Kumar and Panneerselvam (2017) developed an efficient genetic algorithm for the time dependent vehicle routing problem with time windows. They used random sequence insertion-based crossover in their study. They compared the results by using a complete factorial experiments with the two factors namely problem size and algorithm, and found that there are significant differences among the algorithms in terms of the number of vehicles utilized and they also found that the proposed algorithm is better than one more existing algorithm in terms of minimizing the number of vehicles utilized. Sivasankaran and Shahabudeen, (2016) developed a set of genetic algorithms for mixed-model assembly line balancing problem with original task times of models using three crossover methods, viz. cyclic crossover method, forward crossover method and reverse crossover method. They compared the results by using a complete factorial experiment with three-factor namely problem size, cycle time and algorithm, and found that these factors have effect on the balancing efficiency. Hence, using Duncan's multiple range test, they identified the best genetic algorithm for the mixed model assembly line balancing problem. The optimal results obtained by their mathematical model have been compared with the results of the best algorithm and they found that there are no significant differences among them in terms of balancing efficiency. This proves the fact that the best genetic algorithm developed for the mixed model assembly line balancing problem gives results, which are equal or very closer to the corresponding optimal results.

Though the crossover operators have certain characteristics in terms of binary nature or integer nature, etc., when it is plugged into a genetic algorithm, designing a crossover method for a specific problem requires certain attention and newer thinking in terms of design of the crossover method. Hence, in this paper, an attempt has been made to study existing crossover methods used in genetic algorithms and at the end a new crossover method, which is suitable for solving heterogeneous vehicle routing problem with vehicle capacities is proposed.

\section{HETEROGENEOUS VEHICLE ROUTING PROBLEM WITH TIME WINDOWS (HVRPTW)}

The HVRPTW comprises of special variants of the CVRP. It is, therefore, an $N P$-hard combinatorial problem. HVRPTW can be defined as a complete undirected graph $G$ with $(N$ $M)$, where $N$ with $(0--n)$ is a set vertex representing the set of customers with non-negative demands while 0 represents the depot. Each pair of customers $(i, j)$, where $i, j \in N$ and $i \neq j$ i related to a travel time $T_{i j}$ and a distance travelled $D_{i j}$. The service time associated with customers $(i, j)$ is $S_{i}$, and the time window is $(a i, b i)$ to meet the non-negative demand. The element $\mathrm{Si}$ represents the loading or unloading service time at the customer $i$ and $a_{i}$ describes the initial time when it is possible to start the service. If any vehicle arrives at customer $i$ before $a_{i}$, it has to wait there till $a_{i}$ to start servicing that customer. The vehicle must complete the customer service before $b_{i}$. This type of time window constraints is known as a hard time window (Alvarenga et al. 2007). In this problem, each customer has to be served by the unlimited fleet 
of vehicles with different capacities within the time windows associated with them, where the ultimate objective of this problem is to find out the routes for a set of vehicles in order to satisfy some constraints of the CVRP like each route starts and ends at the depot, and such that each customer is visited exactly once by one vehicle, demand of each customer has to be served in the time window associated with it, the total demand of a route does not exceed the capacity of the vehicle assigned to it, and in this process, the total cost is minimized.

\section{GENETIC ALGORITHM (GA)}

The GA is an adaptive heuristic search algorithm, based on the evolutionary ideas of natural selection. The basic concept of the GA is designed by using the theory of "survival of the fittest", developed by Charles Darwin. The solution of the GA is typically involved the same basic steps like representation, selection, recombination (crossover), and mutation. In the first step, the representation of chromosomes represents the substantial structures of a solution. Chromosomes defined as individual members of a population naturally seen by a bit string. A chromosome is a combination of genes. The genes are having the basic features of a solution (Paneerselvam 2016). The selection step involves choosing different chromosomes from the old population for crossover. The step of recombination or reproduction process uses the genes of selected parents to generate offspring for the next generation.

The basic structure of GA is presented below (Chand and Mohanty 2013).

Step 1: Randomly initialize the population.

Step 2: Set the maximum generation.

Step 3: Initialize the generation count as one.

Step 4: Evaluate fitness function of each chromosome in the population

Step 5: While (generation count $<$ maximum generation)

Step 5(a): Sort the chromosomes in ascending order or descending order for minimization or maximization type of objective function, respectively.

Step 5(b): Apply selection procedure to select a set of chromosomes from the population, for the next generation

Step 5(c): Apply genetic operators (crossover and mutation) for the chromosomes of the subpopulation to obtain their offspring.

Step 5(d): Replace the chromosomes of the population by their corresponding offspring.

Step 5(e): generation count $=$ generation count +1

\}.

Step 6: Treat the top most chromosome of the last generation as the solution for implementation.

\section{TYPES OF CROSSOVER OPERATORS}

Crossover operation is a dynamic step for generating new offspring by combing two or more parent chromosomes. These offspring become parent chromosomes for the next generation. The main objective of the crossover step is to make copies of good solutions while eliminating unfair solutions in a population, in order to keep the population size constant. The main objective of this paper is to study about the most appropriate crossover operators and to propose a new crossover operator to solve VRP and its variants, especially HVRPTW.

Some selected crossover methods presented by various authors to solve HVRPTW are listed below. 
- Best Cost Route Crossover Method

- Sub Route Sequence Crossover Method

- Partially Optimised Cyclic Shift Crossover (POCSX) Method

- Stochastic Partially Optimise Cyclic Shift Crossover Method

- Alternative Edge Crossover Method

- Heuristic Greedy or Random or Probabilistic Crossover Method

- Merge 1 And Merge 2 Crossovers Method

- Random Rail Fence Crossover Method

\subsection{Best Cost Route Crossover (BCRC) Method}

BCRC Method is a problem-specific crossover operator method. First, it was proposed by Ombuki et al. (2006). The main objective of the BCRC method is to minimize the number of vehicles and simultaneously cost, which is associated with the operation of the vehicles subject to satisfying all the constraints. The steps of this crossover method are presented below.

1. Assume two parents, viz. Parents P1 and P2 and two offspring OF1 and OF2 as shown below.

$$
\begin{array}{lccccccccc}
\mathrm{P}_{1}: & 3 & 1 & 7 & 5 & 6 & 4 & 2 & 8 & 9 \\
\mathrm{P}_{2}: & 1 & 9 & 8 & 2 & 4 & 6 & 5 & 7 & 3 \\
\mathrm{OF}_{1}: \text { Null } & & & & & & & & \\
\mathrm{OF}_{2} \text { : Null } & & & & & & & &
\end{array}
$$

2. Randomly select two cut points on $\mathrm{P} 1$ and $\mathrm{P} 2$ for creating substring.

Substring1 Substring $2 \quad$ Substring 3

$$
\begin{array}{lll}
\mathrm{P}_{1}: & 317 & \mid 56 \\
\mathrm{P}_{2}: & 198 & \mid 2465
\end{array}
$$

3. From each parent, a substring is chosen randomly. From the parent $\mathrm{P}_{1}$, the substring consisting of the genes 5 and 6 is chosen and from the parent $\mathrm{P}_{2}$, the substring consisting of the genes 7 and 3 is chosen. Then delete the genes present in the substring of a parent from the substrings of the other parent to create offspring. The resulting partial offspring are as shown below.

$\begin{array}{lll}\text { Substring1 } & \text { Substring } 2 & \text { Substring } 3 \\ \mathrm{OF}_{1}: 1 & 5 & 6 \\ \mathrm{OF}_{2}: 198 & 24 & 4289\end{array}$

4. Placing genes of selected string of one parent in the offspring corresponding to other parent.

Note: In this step, the operator will place the genes of the selected string in the best possible location in the corresponding offspring.

4.1 Placing of the genes of substring3 (7 3) of the parent $P_{2}$ in the partial offspring 1.

4.1.1 Choose any of the genes from the substring (7 3) of the parent $\mathrm{P}_{2}$ randomly.

Let it be the gene 3 . 
4.1.2 For extending the offspring $\mathrm{OF}_{1}$, the feasible locations in it for the gene 3 are as follows. Each of the following locations is a feasible location, because the capacity of the vehicle assigned to that substring is sufficient to take up the load of the new gene, which is to be inserted in it now.

A- Before the gene 1 and after the gene 1 .

B- After the gene 5 .

C- Before the gene 6 and after the gene 6 .

D- After the gene 8 .

E- Before the gene 9 and after the gene 9.

By following the above logic, theextended offspring $\mathrm{OF}_{1}$, which is obtained from the earlier partial offspring 1 is as follows by locating the gene 3 after the gene 8

\begin{tabular}{lllll} 
& Substring 1 & Substring 2 & Substring 3 \\
$\mathrm{OF}_{1}:$ & 1 & 5 & 6 & 428 \\
\hline
\end{tabular}

4.2 Placing on of the genes of substring2 (5 6) of the parent $\mathrm{P}_{1}$ in the partial offspring $2\left(\mathrm{OF}_{2}\right)$. 4.2.1 Choose any of the genes from the substring2 (5 6) of the parent $\mathrm{P}_{1}$ randomly. Let it be the gene 5 .

4.2.2 For extending the partial offspring $\mathrm{OF}_{2}$, the feasible locations in it for the gene 5 in the selected string of the parent $\mathrm{P}_{1}$ are as follows. Each of the following locations is a feasible location, because the capacity of the vehicle assigned to that substring is sufficient to take up the load of the new gene, which is to be inserted in it.

F- Before the gene 9 and after the gene 9.

G- After the gene 8 .

H- Before the gene 7 and after gene 7 .

I- Before the gene 3 and after the gene 3 .

By following the above logic, the extended offspring $\mathrm{OF}_{2}$, which is obtained from the earlier partial offspring 2 is as follows by location it before the gene 9 .

\begin{tabular}{|c|c|c|c|c|c|}
\hline \multicolumn{3}{|c|}{ Substring1 } & \multirow[b]{2}{*}{24} & Substring 2 & \multirow[t]{2}{*}{ Substring 3} \\
\hline $\mathrm{OF}_{2}$ : & 198 & 5 & & 73 & \\
\hline
\end{tabular}

4.3 For modifying latest partial offspring $\mathrm{OF}_{1}$, the feasible locations in it for the gene 7 of the selected string of the parent $\mathrm{P}_{2}$ are as follows. Each of the following locations is a feasible location, because the capacity of the vehicle assigned to that substring is sufficient to take up the load of the new gene, which is to be inserted in it.

J- After the gene 1.

K- After the gene 6 .

L- Before the gene 9 and after the gene 9.

By following the above logic, the modified offspring $\mathrm{OF}_{1}$, which is obtained from the previously formed offspring $\mathrm{OF}_{1}$ is as follows by location the gene 7 before the gene 9 . . 
Substring1 Substring $2 \quad$ Substring 3

$\begin{array}{lllllll}\mathrm{OF}_{1}: & 1 & 56 & 4 & 2 & 8 & 3 \lcm{79}\end{array}$

4.4 For modifying offspring $\mathrm{OF}_{2}$, the feasible location in it for the gene 6 of the selected string of the parent $\mathrm{P}_{1}$ is after the gene 3 in it and accordingly, it is inserted as shown below. The insertion of the gene 6 after the gene 3 is possible, because the vehicle capacity is sufficient to take that node in that substring, which represents a route from the depot to different nodes and back to the depot.

Substring 1

$\mathrm{OF}_{2}$ : $\quad 1598$
Substring 2

73

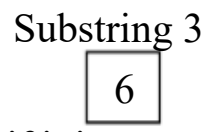

The location of placement of a gene in an offspring is called infeasible if it is not satisfying the constraints like "not meeting the vehicle capacity" or "not satisfying time window" and that is called feasible if it is satisfying all the constraints.

\subsection{Sub Route Sequence Mapped Crossover Method (SRSMXM) Method}

Chand and Mohanty (2013) proposed the problem specific crossover method named as SRSMXM Method. The steps of this crossover method are presented below.

1. Assume that there are two offspring. Initialize the number of sub tours in each of the offspring to 0 as shown below.

Number of sub tours in the offspring $1, \mathrm{NST}_{1}=0$,

Number of sub tours in the offspring $2, \mathrm{NST}_{2}=0$

Input two chromosomes 1 and 2 , which are as shown below.

Chromosome 1: $391-257-864$

Chromosome 2: $152-938-6-47$

2. Form a string of the last nodes of the sub-routes in the chromosome 1, and treat that string as the first sub route of the offspring 1 and initialize the number of sub tours in the offspring 1 to $1\left(\mathrm{NST}_{1}=1\right)$.

(All the constraints need to be satisfied and if some constraints are not satisfied then necessary changes should be made).

3. Form a string of the last nodes of the sub-routes in the chromosome 2, and treat that string as the first sub route of the offspring 2 and initialize the number of sub tours in the offspring 2 to $1\left(\mathrm{NST}_{2}=1\right)$.

(All the constraints need to be satisfied and if some constraints are not satisfied then necessary changes should be made).

The results of Step 2 and Step 3 give partial offspring 1 and partial offspring 2 are as given below.

Offspring1: 174

Offspring 2: 2867 
4. Delete the nodes of the recent sub tour $\left(\mathrm{NST}_{2}{ }^{\text {th }}\right.$ sub tour) of the offspring 2 from the chromosome 1, wherever they occur in it and delete the nodes of the recent sub tour ( $\mathrm{NST}_{1}^{\text {th }}$ sub tour) of the offspring 1 from the chromosome 2, wherever they occur in it. After these deletions, the remaining sub-routes of the two chromosomes are shown below.

$\begin{array}{llll}\text { Chromosome 1: } & 391 & -5 & 4 \\ \text { Chromosome 2: } & 52 & -938 & 6\end{array}$

5. Increment the number of sub tours in the offspring 1 by $1\left(\mathrm{NST}_{1}=\mathrm{NST}_{1}+1\right)$.

6. Form a string of the last nodes of the sub-routes in the chromosome 2, and treat that string as the $\mathrm{NST}_{1}{ }^{\text {th }}$ sub tour of the offspring 1.

7. Increment the number of sub tours in the offspring 2 by $1\left(\mathrm{NST}_{2}=\mathrm{NST}_{2}+1\right)$.

8. Form a string of the last nodes of the sub-routes in the chromosome 1, and treat that string as the $\mathrm{NST}_{2}{ }^{\text {th }}$ sub tour of the offspring 2 .

The results of Step 6 and Step 8 give partial offspring 1 and partial offspring 2 are as given below.

$$
\begin{array}{ll}
\text { Offspring 1: } & 174 \quad 286 \\
\text { Offspring 2: } & 2867-154
\end{array}
$$

9. Delete the nodes of the recent sub tour $\left(\mathrm{NST}_{2}{ }^{\text {th }}\right.$ sub tour) of the offspring 2 from the chromosome 1, wherever they occur in it and delete the nodes of the recent sub tour ( $\mathrm{NST}_{1}^{\text {th }}$ sub tour) of the offspring 1 from the chromosome 2 , wherever they occur in it. After these deletions, the remaining sub-routes of the two chromosomes are shown below.

$$
\begin{array}{lll}
\text { Chromosome 1: } & 39 & \\
\text { Chromosome 2: } & 5 & 93
\end{array}
$$

10. Repeat from Step 5 to Step 9 until all the nodes in both the chromosomes are deleted. The final offspring are as given below. One can see the routes in each of the offspring. Each string in each of the offspring is to be prefixed with depot and also suffixed with depot to for the corresponding route. .

$$
\begin{array}{llll}
\text { Offspring 1: } 174 & -286 & -53 & 9 \\
\text { Offspring 2: } 2867 & -154 & -9 & 3
\end{array}
$$

\subsection{Partially Optimised Cyclic Shift Crossover (POCSX) Method}

Pierre and Zakaria (2014) presented a systematic crossover approach, which generates the offspring without the need for the subsequent corrective procedure. In POCSX, two types of genes are involved, viz. customer genes and delimiter genes. A customer gene represents a customer node in problem and a delimiter gene represents the starting or ending point of a route.

While constructing a route in the offspring, the following parameters are used.

- Size of pool of potential genes

- Random offset count

- Time window 
The pool of potential genes consists of one gene from parent vector $1\left(\mathrm{PV}_{1}\right)$ and two genes selected with a random offset from the beginning of the patent vector $2\left(\mathrm{PV}_{2}\right)$. If the length of the parent vector is not sufficient to accommodate the offset count, then the two genes from the parent vector 2 will selected in cyclic manner. The time window represents an upper bound on the distance or time of travel for each route in the offspring of the vehicle routing problem

The steps of POCSX method are as follows.

1. Assume two parents $P_{1}$ and $P_{2}$ and their corresponding vectors $P V_{1}$ and $P_{2}$ as shown below. The vector of a parent is a sequence of non-zero genes from left to right of that parent.

$$
\begin{aligned}
& \mathrm{P}_{1}: 0410030250 \\
& \mathrm{P}_{2}: 015200000034 \\
& \mathrm{PV}_{1}: 41325 \\
& \mathrm{PV}_{2}: 152234
\end{aligned}
$$

The costs of edges formed by different pairs of genes out of the genes $0,1,2,3,4$ and 5 are shown in Table 1.

Table 1 Costs of Edges of Network Formed by Genes [ $\left.\mathrm{C}_{\mathrm{ij}}\right]$

\begin{tabular}{|c|l|l|l|l|l|l|}
\hline Genes & $\mathbf{0}$ & $\mathbf{1}$ & $\mathbf{2}$ & $\mathbf{3}$ & $\mathbf{4}$ & $\mathbf{5}$ \\
\hline $\mathbf{0}$ & 0.0 & 2.5 & 1.0 & 3.0 & 1.5 & 3.0 \\
\hline $\mathbf{1}$ & 2.5 & 0.0 & 2.0 & 1.0 & 1.5 & 3.5 \\
\hline $\mathbf{2}$ & 1.0 & 2.0 & 0.0 & 2.5 & 1.0 & 2.0 \\
\hline $\mathbf{3}$ & 3.0 & 1.0 & 2.5 & 0.0 & 3.0 & 1.0 \\
\hline $\mathbf{4}$ & 1.5 & 1.5 & 1.0 & 3.0 & 0.0 & 3.0 \\
\hline $\mathbf{5}$ & 3.0 & 3.5 & 2.0 & 1.0 & 3.0 & 0.0 \\
\hline
\end{tabular}

In the Table $1, C_{i j}$ represents the cost of the edge formed from the gene $i$ to the gene $j . I t$ is assumed that the upper bound of time window for all the customers is 4.0 time units. The demand of the customer and the capacity of the vehicles are not taken into consideration.

1. Place the zero value gene as the first gene of the offspring and the corresponding total cost of the partially formed route is 0 .

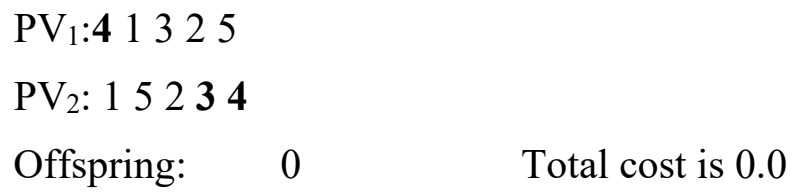

2. First gene (4) of $\mathrm{PV}_{1}$ is added to the pool of potential genes. Here, the maximum capacity of the pool is 3 , which is the number of customers in a route. So, the next two values should be added from the right hand side of $\mathrm{PV}_{2}$. These two values are 4 and 3 . But, the value 4 is already taken in the pool. So, the gene 3 only is added to the pool. The pool is now having two values, which are 4 and 3 . Based on the Table 1, the cost of each of the edges, viz. 0 to 4 and 0 to 3 from the gene 0 is as given below.

$$
\mathrm{C}_{04}=1.5
$$


Study of Crossover operators of Genetic Algorithm\& Development of New Crossover Operator To

Solve Heterogeneous Vehicle Routing Problem with Time Windows

$$
\mathrm{C}_{03}=3
$$

Since the edge $\mathrm{C}_{04}$ has the least cost, the gene 4 is added to the offspring as shown below and the corresponding current cost of the route is increased by 1.5 units. Hence the total cost of the partial offspring developed is 0 plus 1.5 , which is 1.5 units.
Offspring-:
04
Total cost is 1.5

Now, the gene 4 is to be removed from the vectors $P V_{1}$ and $P_{2}$. The resultant vectors after the removal of this gene are as shown below.

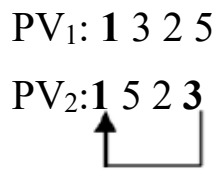

3. The first gene of $\mathrm{PV}_{1}$ is 1 . This is added to the pool of potential genes and the next two values should be added from $\mathrm{PV}_{2}$. The two values, which are taken from the $\mathrm{PV}_{2}$ should be in the cyclic order which are 3 and 1 . Because the gene 1 is already present in the pool of genes, the gene 3 only added to that pool. So, now this pool contains the genes 3 and 1 .

Based on the Table 1, the cost of each of the edges, viz. 4 to 3 and 4 to 1 from the gene 4 is as given below.

$$
\begin{aligned}
& \mathrm{C}_{41}=1.5 \\
& \mathrm{C}_{43}=3
\end{aligned}
$$

Since the edge $\mathrm{C}_{41}$ has the least cost, the gene 1 is added to the offspring as shown below and the corresponding current cost of the route is increased by 1.5 units. Hence the total cost of the partial offspring developed is 1.5 plus 1.5 , which is 3 units.
Offspring-:
$\begin{array}{lll}0 & 4 & 1\end{array}$
Total cost is 3 .

Now, the gene 1 is to be removed from the vectors $P V_{1}$ and $P_{2}$. The resultant vectors after the removal of this gene are as shown below.

$$
\begin{aligned}
& \mathrm{PV}_{1}: 325 \\
& \mathrm{PV}_{2}: 523
\end{aligned}
$$

4. The first gene of $\mathrm{PV}_{1}$ is 3. This is added to the pool of potential genes and the next two values should be added from $\mathrm{PV}_{2}$. The two values, which are taken from the $\mathrm{PV}_{2}$ in cyclic order which are 5 and 2 are added to that pool. So, now this pool contains the genes 3,5 and 2 .

Based on the Table 1, the cost of each of the edges, viz. 1 to 3,1 to 5 and 1 to 2 from the gene 1 is as given below.

$$
\begin{aligned}
& \mathrm{C}_{13}=1 \\
& \mathrm{C}_{15}=3.5 \\
& \mathrm{C}_{12}=2
\end{aligned}
$$

Since the edge $\mathrm{C}_{13}$ has the least cost, the gene 3 is added to the offspring as shown below and the corresponding current cost of the route is increased by 1 units. Hence the total cost of the partial offspring developed is 3 plus1, which is 4 units. Since the total cost is equal to the 
value of the time window, which is 4 , the current route is terminated and 0 is suffixed at the end to indicate the depot.

$$
\text { Offspring-: } \quad \begin{array}{llllllll}
0 & 4 & 1 & 30 & \text { Total cost is } 4 .
\end{array}
$$

Now, the gene 3 is to be removed from the vectors $\mathrm{PV}_{1}$ and $\mathrm{PV}_{2}$. The resultant vectors after the removal of this gene are as shown below.

$$
\begin{aligned}
& \mathrm{PV}_{1}: 25 \\
& \mathrm{PV}_{2}: 52
\end{aligned}
$$

5. Second route is to be created in the offspring from the depot indicated by the last gene in it with the total cost of 0 as shown below

$$
\text { Offspring-: } \quad \begin{array}{lllllllll}
0 & 4 & 1 & 3 & 0 & \text { Total cost is .0 }
\end{array}
$$

The first gene of $P V_{1}$ is 2 . This is added to the pool of potential genes and the next two values should be added from $\mathrm{PV}_{2}$. The two values, which are taken from the $\mathrm{PV}_{2}$ in cyclic order which are 2 and 5 are to be added to that pool. Since, the gene 2 is already in the pool of potential genes, the gene 5 only is added to that pool. So, this pool contains the genes 2 and 5 .

Based on the Table 1, the cost of each of the edges, viz. 0 to 2 and 0 to 5 from the gene 0 is as given below.

$$
\begin{aligned}
& \mathrm{C}_{02}=1 \\
& \mathrm{C}_{05}=3
\end{aligned}
$$

Since the edge $\mathrm{C}_{02}$ has the least cost, the gene 2 is added to the offspring as shown below and the corresponding current cost of the route is increased by 1 units. Hence the total cost of the partial offspring developed is 0 plus 1 , which is 1 unit.

$$
\text { Offspring-: } \quad \begin{array}{llllllllll}
0 & 4 & 1 & 3 & 0 & 2 & \text { Total cost is } 1 .
\end{array}
$$

Now, the gene 2 is to be removed from the vectors $\mathrm{PV}_{1}$ and $\mathrm{PV}_{2}$. The resultant vectors after the removal of this gene are as shown below.

$$
\begin{array}{ll}
\mathrm{PV}_{1}: & 5 \\
\mathrm{PV}_{2}: & 5
\end{array}
$$

6. Since the gene 5 is the only gene in each of the parent vectors, the pool of potential gene contains only one gene.

Based on the Table 1, the cost of the edge 2 to 5 from the gene 0 is 2 .

Since the gene 5 is the only gene in the pool of potential genes, it is added to the offspring as shown below and the corresponding current cost of the route is increased by 2 units. Hence the total cost of the partial offspring developed is 1 plus2, which is 3 unit.
Offspring-:
$\begin{array}{lllllll}0 & 4 & 1 & 3 & 0 & 2 & 5\end{array}$
Total cost is 3 .

Now, the gene 5 is to be removed from the vectors $\mathrm{PV}_{1}$ and $\mathrm{PV}_{2}$. The resultant vectors after the removal of this gene are as shown below.

$$
\begin{aligned}
& \mathrm{PV}_{1} \text { : Null } \\
& \mathrm{PV}_{2} \text { : Null }
\end{aligned}
$$

Since, each of the parent vectors is a null set, the construction of the second route comes to an end and hence the gene corresponding to the depot, which is 0 is suffixed at the end of the earlier partial offspring as shown below for which the total cost is 3 .
Offspring-:
$\begin{array}{llll}0 & 4 & 1 & 3\end{array}$
250
Total cost is 3 . 
Based on the two parents and their parent vectors, with respect to the parent vector 1 , the complete offspring contains two routes, viz. 04130 and 0250 .

\section{4. Stochastic Partially Optimised Cyclic Shift Crossover (SPOCSX) Method}

Pierre and Zakaria (2017) presented the SPOCSX method. The working process of this method is based on POCSX method. However, POCSX method matches the hill-climbing instrument by successively adding genes whose addition results in low-cost edges, while in SPOCSX method, hill-climbing is ruled by probabilistic rules. The main aim of using probabilistic rules in this method is to overcome the limitations of the greedy construction algorithm shown by POCSX. The rules dictate at what point in time the algorithm should emulate the hill-climbing by adding the lowest cost gene or the highest cost gene in order to give the lowest cost gene a chance to fit in another route.

\subsection{Alternative Edge Crossover (AEX) Method}

The AEX operator works as a directed cycle of arcs. The offspring cycle is formed by choosing arcs from the parents $\mathrm{P}_{1}$ and $\mathrm{P}_{2}$ alternatively, with some additional random selections if there arises any infeasibility. The steps of AEX method are as follows.

1. Assume two parents, viz. $P_{1}$ and $P_{2}$ and a null offspring as shown below.

$$
\begin{aligned}
& \mathrm{P}_{1}: 517849623 \\
& \mathrm{P}_{2}: 362519847 \\
& \text { OF: Null }
\end{aligned}
$$

2. The procedure of constructing offspring will start by choosing the arc 5 O 1 fon the $\mathrm{P} 1$ as it is as the first arc. So the construction of the offspring is initialised as shown below.

$$
\text { OF:(5 } 1
$$

3. Next, the arc that goes out from the gene 1 in $\mathrm{P}_{2}$ is added. That is $1 \subset 9$. Sote extended offspring is as shown below.

$$
\text { OF: (5 } 19
$$

4. Next, the arc that goes out from the gen 9 in $\mathrm{P}_{1}$ is added, i.e. $9 \circlearrowleft 6$. So the offingis modified to the following form.

$$
\text { OF: (5 } 196
$$

5. After performing few steps, a partial offspring will be as shown below.

$$
\text { OF: (5 } 199623
$$

6. The arc going out from 3 should be chosen from $\mathrm{P}_{2}$, which is $3 \bigcirc 6$. But such duis not desirable, since it will form a sub tour.

The construction of the offspring should such that there are no sub tours, which means that the given nodes should be placed in a randomized manner in the offspring.

To avoid this situation, one of the remaining unselected genes is chosen randomly, say 7 . So the offspring is modified to the following form. 
OF: (5 $19962237 \ldots$ _)

7. Now, again the same step can be followed for selecting the arc $7 \subset 8$ from $\mathrm{P}_{1} \mathbf{d}$ then $8 \bigcirc 4$ from $\mathrm{P}_{2}$. At the end, the complete offspring is as follows.

OF: (5 1966237784$)$

\subsection{Heuristic Crossover (HGreX) Method}

The HGreX operator also works in a similar pattern like AEX method, but slight changes exist in it to form the offspring. Here, the offspring is constructed by selecting each gene, which is cheaper among the two parents. If any infeasibility arises in creating the offspring, then some random selection is done according to the situation. The steps of HGreX method are as follows.

1. Assume two parents, viz. $\mathrm{P}_{1}$ and $\mathrm{P}_{2}$ and also assume the associated costs (distances) with the arcs of each of the parents as shown below.

$$
\begin{aligned}
& \mathrm{P}_{1}: \mathrm{c} 51=2, \mathrm{c} 17=2, \mathrm{c} 78=6, \mathrm{c} 84=8, \mathrm{c} 49=3, \mathrm{c} 96=6, \mathrm{c} 62=4, \mathrm{c} 23=4, \mathrm{c} 35=3 \\
& \mathrm{P}_{2}: \mathrm{c} 36=6, \mathrm{c} 62=4, \mathrm{c} 25=2, \mathrm{c} 51=2, \mathrm{c} 19=6, \mathrm{c} 98=8, \mathrm{c} 84=8, \mathrm{c} 47=3, \mathrm{c} 73=5
\end{aligned}
$$

The procedure of constructing offspring will start by randomly choosing a gene, say 5 . The arc which is associated with the gene 5 is $5 \circlearrowleft 1$ in $\mathrm{P}_{1}$ as well as from $\mathrm{P}_{2}$. The cost which associated with it is the same. So, any one of the arc can be selected and let it be the arc $5(\mathbf{O}$ Based on this selection, the offspring is initialised by including this arc in that offspring as shown below.

$$
\text { OF: }(51
$$

2. The arcs which are going out from the gene 1 are as listed below.

$1 \bigodot 7$ in $\mathrm{P}_{1}$ with associat ed cost of 2 units.

$1 \bigodot 9$ in $\mathrm{P}_{2}$ with associated cost of 6 units.

Now the cheapest among both the genes, viz. 7 and 9 is chosen to augment in the offspring, which is the gene 7 . The extended offspring with the inclusion of this gene is as shown below.

$$
\text { OF: (5 } 17
$$

3. The arcs which are going out from the gene 7 are listed below.

$7 \bigcirc 8$ inP $P_{1}$ with associat ed cost of 6 units.

$7 \bigcirc 3$ in $\mathrm{P}_{2}$ with associat ed cost of 5 units.

Now the cheapest among both the genes, viz. 8 and 3 is chosen and added to the offspring as shown below.

$$
\text { OF: (5 } 1773
$$

4. The arcs which are going out from the gene 3 are as listed below.

$3 \circlearrowright 5$ in $\mathrm{P}_{1}$ with associated cost of 3 units,

$3 \circlearrowright 6$ inP $_{2}$ with associat ed cost of 6 units

Now, the cheapest among both the genes, viz. 5 and 6 is the gene 5. But, the arc $3 \subset 5$ is infeas ible, because the gene 5 is already included in the offspring. Sote next gene 6 is added to the offspring as shown below. 
Study of Crossover operators of Genetic Algorithm\& Development of New Crossover Operator To

Solve Heterogeneous Vehicle Routing Problem with Time Windows

$$
\text { OF: (5 } 1736
$$

5. The arcs which are going out from gene 5 are as listed below.

$$
\begin{aligned}
& 6 \circlearrowright 2 \text { in } P_{1} \text { with associated cost of } 4 \text { units } \\
& 6 \circlearrowright 2 \text { in } P_{2} \text { with associated cost of } 4 \text { units }
\end{aligned}
$$

Now the cost associated with both the arcs are the same as well as the destination genes of both arcs are the same. Hence, the gene 2 is randomly selected and added to the offspring as shown below.

$$
\text { OF: (5 } 1773662
$$

6. The arcs which are going out from gene 2 are listed below.

$2 \circlearrowright 3$ in $\mathrm{P}_{1}$ with associated cost of 4 units

$2 \circlearrowright 5$ in $\mathrm{P}_{2}$ with associated cost of 2 units

Now the cheapest among both of them is chosen but both the arcs are infeasible, because the genes 3 and 5 are already present in the partial offspring. To avoid this situation, the operator will select an arc fromamong the randomly generated feasible arcs, viz. which are $2(\mathbf{O}), 2(\mathbb{8}$ and $2 \circlearrowleft$ 9. Let that arc be $2 \circlearrowleft 8$ and it is added into the offspring as shown below. OF: (5 $\begin{array}{lllllll}5 & 1 & 7 & 3 & 6 & 2 & 8\end{array}$

7. The arcs which are going out from gene 8 are as listed below.

$$
\begin{aligned}
& 8 \bigcirc 4 \text { in } P_{1} \text { with assoc iated cost of } 8 \text { units } \\
& 8 \bigcirc 4 \text { in } \mathrm{P}_{2} \text { with associat ed cost of } 8 \text { units }
\end{aligned}
$$

Now the cost associated with both are the same as well the destination genes of both the arcs are the same. So, the gene 4 is added to the offspring as shown below.

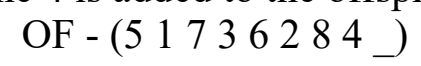

8. The arcs which are going out from gene 4 are as listed below.

a) $4 \circlearrowright 9$ in $P_{1}$ with associated cost of 3 units

b) $4 \bigodot 7$ in $\mathrm{P}_{2}$ with associated cost of 3 units (Infeasible arc)

Since $4 \bigcirc 9$ is the only left out arc, the gene 9 is added to the offspring as bon below.

$$
\text { OF - (5 } \left.1573 \begin{array}{lllll} 
& 6 & 2 & 8 & 4
\end{array}\right)
$$

The heuristic random crossover (HRndX) method or heuristic probabilistic crossover (HProX) method, operators are variants of HGreX method. These operators follow the same steps of HGreX method, but follow different norms for selecting an arc. The HGreX method follows the greedy approach and always selects the cheapest cost, whereas HRndX method and HProX method select the arc randomly. The HRndX method selects an arc with equal probability and the HProX method selects the cheapest arc. For the same parents P1 and P2 utilised in HGreX method, HRndX method or HProX method will generate a different offspring when compared to the offspring generated by the HGreX method. 


\subsection{Merge $1\left(\mathrm{MX}_{1}\right)$ and Merge 2 Crossover $\left(\mathrm{MX}_{2}\right)$ Method}

Blanton and Wainwright (1993) presented MX1 and MX2 crossover operators for MultiObjective Genetic Algorithms (MOGA). These operators are based on the global precedence among the genes, which is independent of any of the chromosomes. The working logic of MX1 and $\mathrm{MX}_{2}$ crossover methods are as follows.

\section{MX $_{1}$ Crossover Method}

The workings of MX1 crossover method are explained below.

1. Assume two chromosomesas shown below.

Chromosome 1:2561073849

Chromosome 2: 4169382057

Let the Global Preceding Vector (GPV) be 0123456789 .

2. The first genes of the Chromosome 1 and the Chromosome 2 are considered for creating an offspring. In GPV, the first gene on the Chromosome 1 is found to occur earlier than that of the Chromosome 2. So, the fist gene on the chromosome 1 is included in the first available gene position of the offspring, and simultaneously the first gene on chromosome 2 is swapped with the first gene on the Chromosome 1.The resultant chromosomes and the offspring 2 are as shown below.

Chromosome 1:2561073849

Chromosome 2: 2169382057

Offspring: 2

3. The $2^{\text {nd }}$ gene of the Chromosome 2occurs earlier than the $2^{\text {nd }}$ gene of the Chromosome1 in the GPV. Hence, the $2^{\text {nd }}$ gene of the Chromosome 2 is included in the first available gene position of the offspring 2 . Simultaneously, the $2^{\text {nd }}$ gene of the Chromosome 1 is swapped with the $2^{\text {nd }}$ gene of the Chromosome 2. The resultant chromosomes and the offspring 2 are as follows. .

Chromosome 1:2161073849

Chromosome 2: 2169382057

Offspring: 21

4. After repeating the above procedure till all the genes of the offspring 2 are filled, the final offspring 2 will be as given below.

Offspring 2: 165073489

\section{$\mathrm{MX}_{2}$ Crossover Method}

The working of $\mathrm{MX}_{2}$ crossover operator is based on merge algorithm, which merges two small vectors.

1. Assume two chromosomes as shown below.

Chromosome 1:2561073849

Chromosome 2: 4169382057

Let the Global Preceding Vector (GPV) be 0123456789. 
2. In GPV, the $1^{\text {st }}$ gene on the Chromosome 1 occurs earliest than the $1^{\text {st }}$ gene on chromosome 2. So the $1^{\text {st }}$ gene on the Chromosome 1, which 2 is included in the first gene position of offspring 2. Simultaneously, this gene 2 is removed from both the chromosomes. The resultant chromosomes and the offspring 2 are as given below.

Chromosome 1: X 561073849

Chromosome 2: $416938 \times 057$

Offspring: 2

3. In GPV, the first undeleted gene from left of the Chromosome 2 which is the gene 4 , occurs earlier than the first undeleted gene from left of the Chromosome 1, which is the gene 5 . So, include the gene 4 in the second position of the offspring 2 and simultaneously remove the gene 4 from both the chromosomes. The resultant chromosomes and the offspring 2 are as given below.

Chromosome 1: X $1610738 \times 9$

Chromosome 2: X $16938 \times 057$

Offspring- 24 X XXXXXXX

4. After repeating the above procedure till all the genes of the offspring 2 are filled, the final offspring 2 will be as given below.

Offspring 2:2415607389.

\subsection{Random Rail Fence Crossover Method}

Sivasankaran and Shahabudeen (2016) proposed reflection crossover method for mixedmodel assembly line balancing problem, which is renamed as random rail fence crossover method in this paper. The steps of this method are presented below.

1. Assume two parents, $\mathrm{P}_{1}$ and $\mathrm{P}_{2}$ as shown below.

$$
\begin{aligned}
& P_{1}=\left(\begin{array}{lllllll}
1 & 2 & 3 & 4 & 6 & 7 & 8
\end{array}\right)
\end{aligned}
$$

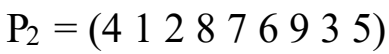

2. Copy the first gene from $P_{1}$ into an offspring. After performing this step, the partially constructed offspring will be as follows.

$$
\text { Offspring }=(1
$$

3. Next, select the gene from $\mathrm{P}_{2}$ at the same position of 1 which is 4 . Copy this gene into offspring but at the position where it is present in $\mathrm{P}_{1}$. The resulting offspring is as given below.

$$
\text { Offspring }=\left(\begin{array}{lll}
1 & -
\end{array}\right.
$$

4. Next, select the gene from $\mathrm{P}_{2}$ at the same position of 4 which is 8 . Copy this gene into the offspring but at the position where it is present in $\mathrm{P}_{1}$. The resulting offspring is as given below.

$$
\text { Offspring }=\left(\begin{array}{ll}
1-{ }_{-}--{ }_{-} \_
\end{array}\right)
$$

5. After performing with two more steps, the resulting offspring will be as follows.

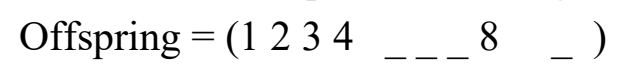


6. Now, the last gene which was copied is 2 at the gene position 2. So, the next choice should be the gene from $\mathrm{P}_{2}$ at the same position where it is present, which is 1 . But, 1 is also not a feasible choice because it is already present in the offspring. In this case, only the parent $\mathrm{P}_{2}$ is used to complete the offspring. All remaining positions of the offspring should be filled by the genes from $\mathrm{P}_{2}$ at their corresponding positions. Thus, the final offspring is as shown below.

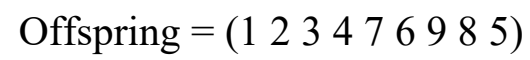

\section{PROPOSED CROSSOVER OPERATOR}

On the basis of literature reviewed, it is found that the traditional crossover methods like PMX, AEX, OX, MX1 and MX2 are not very conducive to optimize the problem of HVRPTW. So, in this paper, an attempt has been made to solve HVRPTW problem based on a practical crossover method. The steps of the proposed crossover method are as follows.

1. Assume two parent chromosomes, viz. $\mathrm{P}_{1}$ and $\mathrm{P}_{2}$, each with a gene size of 9 as shown below.

$$
\begin{aligned}
& P_{1}: 317564289 \\
& P_{2}: 198246573
\end{aligned}
$$

2. Assume six available vehicles, A, B, C, D, E and F with different capacities as shown below.

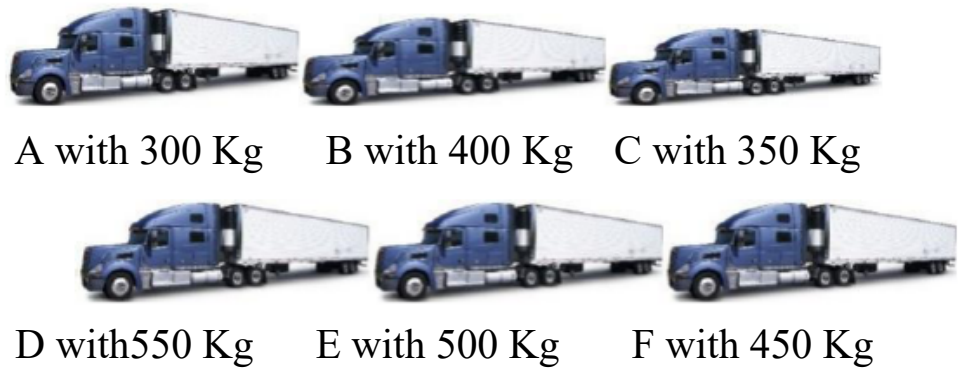

3. Randomly select two cut points, viz. gene position 4 and gene position 5on P1 and gene position 4 and gene position 7 on $\mathrm{P}_{2}$ for creating the sub routes. The sub-routes of $\mathrm{P}_{1}$ and $\mathrm{P}_{2}$ are as shown below.

\subsection{From $\mathrm{P}_{1}$}

$\begin{array}{ccl}\text { Sub route 1 } & \text { Sub route 2 } & \text { Sub route 3 } \\ 317 & 56 & 4289\end{array}$

3.2 From $\mathrm{P}_{2}$

The Sub route 1 Sub route $2 \quad$ Sub route 3

$198 \quad 246573$

3.3 The configuration of $\mathrm{P}_{1}: \quad 317 \quad 56 \quad 4289$

3.4 The configuration of $\mathrm{P}_{2}$ : $198 \quad 246573$

3.5 Assume the demand values of the sub routes as follows.

Parent P1:

The demand for the Sub route 1, "3 17 "is380 Kg

The demand for the Sub route 2, "5 6 "is $350 \mathrm{Kg}$

The demand for the Sub route 3, "4 289 " is $420 \mathrm{Kg}$ 
Parent $\mathrm{P}_{2}$ :

The demand for the Sub route 1, "1 98 ", is $370 \mathrm{Kg}$

The demand for the Sub route 2, "2 465 " is $370 \mathrm{Kg}$

The demand for the Sub route 3, "7 3" is $230 \mathrm{Kg}$.

4. In this step, select one sub route randomly from each parent and simultaneously remove these from opposite parent. Suppose the sub route 2 that is with genes 5 and 6 , is selected from $P_{1}$ and sub route 3 that is with genes 7 and 3 is selected from $P_{2}$. After performing this step new offspring will be as follows.

Offspring 1:1564289

Offspring 2: 1982473

5. Assume the demands of offspring as shown below.

Offspring1: 1 with $150 \mathrm{~kg}, 56$ with $350 \mathrm{~kg}$ and 4289 with $420 \mathrm{~kg}$

Offspring2: 198 with $370 \mathrm{~kg}, 24$ with $250 \mathrm{~kg}$ and 73 with $230 \mathrm{~kg}$

6. Assume the demands of the removed genes as given below. 5 with $50 \mathrm{Kg}$, 6with $70 \mathrm{Kg}$, 7with $80 \mathrm{Kg}$ and 3with $150 \mathrm{Kg}$.

7. Since each chromosome should contain all the genes, the next step is to put the removed genes into the best possible places in the corresponding offspring. For this, some basic steps are to be followed, which are as presented below.

7.1 Arrange the available heterogeneous vehicle in increasing order according to their capacity. Accordingly, the new arrangement of the vehicle is as follows:

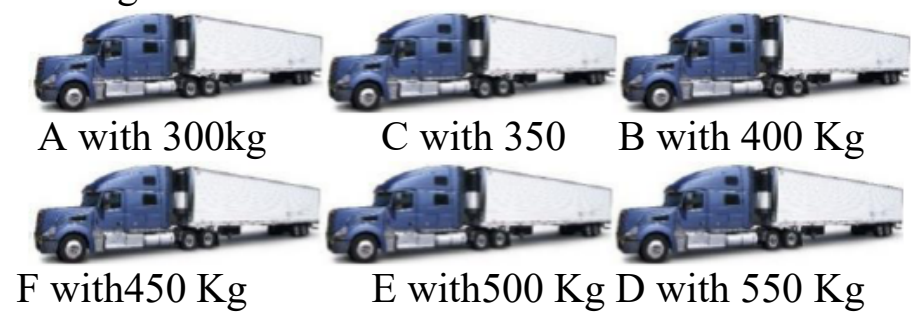

7.2. Arrange the substrings of each offspring in the increasing order of their demand values as shown below.

$$
\begin{array}{ll}
\text { Offspring 1: } 1 \text { with } 150 \mathrm{Kg} & 56 \text { with } 350 \mathrm{Kg} \quad 4289 \text { with } 420 \mathrm{Kg} \\
\text { Offspring 2: } 3 \text { with } 50 \mathrm{~kg} & 247 \text { with } 250 \mathrm{Kg} 198 \text { with } 370 \mathrm{Kg}
\end{array}
$$

7.3. Insert the removed gene 3 and 7 at the end of each sub route of each offspring and check the resultant demand of that sub route.

The first check is with the gene 3 as shown below by suffixing at the end of each sub route of the offspring 1.

13 with $300 \mathrm{Kg}, 5 \quad 6 \quad 3$ with $500 \mathrm{Kg}$ then $4 \quad 2 \quad 8 \quad 9 \quad 3$ with $570 \mathrm{Kg}$

Next check is with the gene 7 as shown below by suffixing at the end of each sub route of the offspring 1.

17 with $230 \mathrm{Kg}, 5 \quad 6 \quad 7 \quad$ with $430 \mathrm{Kg}$ then $\quad \begin{array}{llllll}4 & 2 & 8 & 9 & 7 & \text { with } 500 \mathrm{Kg}\end{array}$

7.4. Now match sub route with the vehicle capacity and find out the best possible way for inserting the removed genes. If the gene 7 is put in the first sub route, then the demand for that sub route will be $230 \mathrm{Kg}$, but there is no vehicle, which is having a capacity of $230 \mathrm{Kg}$. So, the gene 3 is inserted in the sub route 1. Finally, the partially formed offspring 1 is as given below. Offspring 1: 13 with $200 \mathrm{Kg} 56$ with $350 \mathrm{Kg} 4289$ with $420 \mathrm{Kg}$ 
7.5. Now for the next gene 7, there are only two possible ways of inserting in the offspring 1 , which are inserting in the sub route 2 and in the sub route 3 as shown below.

567 with $430 \mathrm{Kg}$ and 42897 with $500 \mathrm{Kg}$.

7.6. Next, match the demand of the sub-routes with the vehicle capacities. Now, the demands of the sub routs can be fulfilled by the available two vehicles, viz. vehicle F with $450 \mathrm{Kg}$ and vehicle $\mathrm{E}$ with $500 \mathrm{Kg}$. Use of vehicle $\mathrm{F}$ satisfies the demand of sub route 2, but it is not possible to utilize the full capacity $(450 \mathrm{Kg})$ of that vehicle. So, the gene 7 is put in the sub route 3 and the vehicle $\mathrm{E}$ which is having a capacity of $500 \mathrm{Kg}$ is used. By this method, the full capacity of the vehicle is utilized in order to satisfy all the constraints. The new offspring as per this allocation is as follows.

Offspring 1: $1 \quad 3 \quad 5 \quad 6 \quad 42897$

7.7. Now, the removed genes 5 and 6 are inserted in each position of the offspring 2 and the demand of each sub route is checked as shown below.

The first check is with the gene 5 as shown below.

35 with $100 \mathrm{Kg}, 2475$ with $300 \mathrm{Kg}$ and 1985 with $420 \mathrm{Kg}$

Next check is with the gene 6 as shown below.

6 with $120 \mathrm{Kg}, 2476$ with $320 \mathrm{Kg}$ and 1986 with $440 \mathrm{Kg}$

8. Now, match the sub-routes with the vehicle capacities and find out the best possible way for inserting the removed gene.

If the gene 5 is put in the first sub route of offspring 2, then the demand of that sub route will be $100 \mathrm{Kg}$. But, there is a vehicle with a capacity of $100 \mathrm{Kg}$. So, the gene 6 is inserted in the sub route 1 of offspring 2 . The demand for this sub route becomes $120 \mathrm{Kg}$. Here also, there is no vehicle with a capacity of $120 \mathrm{Kg}$.

Next, the $2^{\text {nd }}$ sub route of the offspring 2 is cheeked. If the gene 5 is put in the sub route 2 of the offspring 2, then the demand of that sub route will become $300 \mathrm{Kg}$.

9. Now the operator can satisfy this demand by using vehicle A with the capacity of $300 \mathrm{~kg}$. So the new offspring will be as follows.

Offspring 2: $3 \quad 247 \quad 5 \quad 189$

10. Now, the gene 6 is put in the sub route 3 of the offspring 2, which results with a demand of this sub route as $440 \mathrm{Kg}$. But, there is no vehicle with the capacity of 440 $\mathrm{Kg}$. But, the vehicle $\mathrm{F}$ has the capacity of $450 \mathrm{Kg}$, which is closer to $440 \mathrm{Kg}$. So, the gene 6 is put in the sub route 3 of the offspring 2. The new offspring will be as shown below.

Offspring 2: $3 \quad 2475 \quad 1986$

\section{CONCLUSION}

The HVRPTW is a combinatorial and NP-hard problem. So, the usage of meta-heuristics like GA is inevitable.. Crossover is the main step of GA which resolves the effective implementation of GA to HVRPTW. In this article, an attempt has been made to study various prominent crossover operators available in the literature. Then, a new crossover operator for solving the HVRPTW problem is presented. The proposed crossover method is illustrated with a numerical example. There were many crossover operators available in the literature, but all of them may not fit to the particular problem which is used for the study. Therefore, this paper is limited to study select crossover operators, which are relevant for the HVRPTW problem 
Study of Crossover operators of Genetic Algorithm\& Development of New Crossover Operator To Solve Heterogeneous Vehicle Routing Problem with Time Windows

\section{REFERENCE}

[1] Afshar-Nadjafi, B., \& Afshar-Nadjafi, A. (2014). A constructive heuristic for timedependent multi-depot vehicle routing problem with time-windows and heterogeneous fleet. Journal of King Saud University - Engineering Sciences.

[2] Alvarenga, G.B. Mateus, G.R., Tomi G. de, (2007) A genetic and set partitioning two-phase approach for the vehicle routing problem with time windows, Computers \& Operations Research. Vol.-34 pp.1561-1584

[3] Amador-Fontalvo, Jaime \&Paternina-Arboleda,Carlos\& Montoya-Torres, Jairo. (2014). Solving the heterogeneous vehicle routing problem with time windows and multiple products via a bacterial meta-heuristic. International Journal of Advanced Operations Management. Vol.-6, pp.81 - 100.

[4] Anand, E., Panneerselvam, R., (2016) A study of crossover operators for genetic algorithm and proposal of a new crossover operator to solve open shop scheduling problem, American Journal of Industrial and Service Management. Vol-6, No.6, pp.774-789.

[5] B. Ombuki, B. Ross, F. Hanshar, (2006) Multi-objective genetic algorithm for vehicle routing problem with time windows, Applied Intelligence.Vol.24, pp.17-30.

[6] Berger, M. Barkaoui (2004), a parallel hybrid genetic algorithm for the vehicle routing problem with time windows. Computers and Operations Research. Vol- 31 (12) , pp. 20372053

[7] Blanton Jr. Joe L. and Wainwright Roger L. (1993). Multi Vehicle Routing with Time and Capacity Constraints using Genetic Algorithm. Proceedings of the fifth international conference on Genetic Algorithm (ICGA-93), Stephanie Forest, Editor, Morgan Kaufmann Publisher, 1993, pp. 452-459.

[8] Chand, P. and Mohanty,J.R. (2013) A Multi-objective Vehicle Routing Problem using Dominant Rank Method. International Journal of Computer Applications. pp. 29-34.

[9] Chand, P. and Mohanty,J.R. (2013) Real-Time Vehicle Routing Problem With Time Window Simultaneously Delivery Products And Pick Up Wastage Materials With Proposed Master-Slave Genetic Algorithm. International Journal of Computer Applications.Vol.72 No.19, pp39-46.

[10] Chand, P. and Mohanty,J.R. (2013) Solving Vehicle Routing Problem With Proposed NonDominated Sorting Genetic Algorithm And Comparison With Classical Evolutionary Algorithms. International Journal of Computer Applications. Vol 69- No.26 34-41.

[11] Dantzig, G. and Ramser, J. (1959) The Truck Dispatching Problem. Management Science. Vol.6, pp. 80-91.

[12] De, J. J., Carlos, C., \& Montoya Torres, V. C. J. R. (2013). A two-pheromone trail ant colony system tabu search approach for the heterogeneous vehicle routing problem with time windows and multiple products, J Heuristics. Vol.19, pp.233-252

[13] Dondo, R., \& Cerda, J. (2007). Discrete Optimization a cluster-based optimization approach for the multi-depot heterogeneous fleet vehicle routing problem with time windows, European Journal of Operational Research. Vol.176, pp.1478-1507.

[14] Goldberg, D. E. \&Lingle, Jr., R. (1985). Alleles, Loci and the TSP. In Grefenstette, J. J. (ed.) Proceedings of the First International Conference on Genetic Algorithms and Their Applications, pp.154-159. Hillsdale, New Jersey: Lawrence Erlbaum

[15] Golden, B., A. Assad, L. Levy and F.G. Gheysens, 1984. The fleet size and mix vehicle routing problem. Computer and Operation Research. Vol.-11 pp.49-66.

[16] Jabali, O., \&Laporte, G. (2016). Thirty years of heterogeneous vehicle routing, European Journal of Operational Research.Vol-249 pp.1-21.

[17] Kabcome P, Mouktonglang T, (2015) Vehicle routing problem for multiple product types, compartments, and trips with soft time windows. International journal of mathematical science doi:10.1155/2015/126754 
[18] Muthusamy, Aravendan and Panneerselvam, Ramasamy. (2015). Development and Comparison of Hybrid Genetic Algorithms for Network Design Problem in Closed-Loop Supply Chain. Intelligent Information Management. 7. 313-338. 10.4236/iim.2015.76025.

[19] Nagle and Paneerselvam (2016), Literature Review on Heterogeneous Vehicle Routing Problem. Conference Proceedings in 3i Intelligence, Innovation and Inclusion - Best Practices for Global Excellence. Editor, ChitraSivasundaram. Kasilingam, B. Rajeshwari,R. Vijay Nicole Imprints Ltd. Chennai PP.-879 to 896.

[20] Nanda Kumar, Suresh \& Panneerselvam, Ramasamy. (2017). Development of an Efficient Genetic Algorithm for the Time Dependent Vehicle Routing Problem with Time Windows. American Journal of Operations Research. 07. 1-25. 10.4236/ajor.2017.71001.

[21] Panneerselvam, R. (2012) Research Methodology. Second Edition, PHI Learning, New Delhi.

[22] Panneerselvam, R. (2016) Design and Analysis of Algorithms. Second Edition, PHI Learning, New Delhi

[23] Pierre, D. M. \&Zakaria, N. 2017. Stochastic partially optimized cyclic shift crossover for multi-objective genetic algorithms for the vehicle routing problem with time-windows. Applied Soft Computing, 3838, 14-28.

[24] PierreD.M., ZakariaN., (2014) Partially optimized cyclic shift crossover for multi-objective genetic algorithms for the multi-objective vehicle routing problem with time-windows, IEEE Symposium on Computational Intelligence in Multi-Criteria Decision-Making, Orlando, FL, USA, December 9-12, pp. 106-115.

[25] Pulji'c, K and Manger, R. (2013) Comparison of eight evolutionary crossover operators for the vehicle routing problem. Journal of Mathematical Communications.Vol-18, pp.359375.

[26] Rachid, Mais\&RamdaneCherif, Wahiba\&Chatonnay, Pascal \& Bloch, Christelle. (2010). A study of performance on crossover and mutation operators for vehicle routing problem. Proceedings of the 3rd International Conference on Information Systems, Logistics and Supply Chain Creating value through green supply chains ILS 2010 - Casablanca (Morocco), April 14-16

[27] Senthilkumar and Narayanan, Sockalingam. (2011). GA Based Heuristic to Minimize Makespan in Single Machine Scheduling Problem with Uniform Parallel Machines. Intelligent Information Management. 3. 204-214. 10.4236/iim.2011.35025.

[28] Sivasankaran P. and Shahabudeen P. M. (2014). Study and Analysis of GA-Based Heuristic Applied to Assembly Line Balancing Problem. Journal of Advanced Manufacturing Systems. 13. 113-131. 10.1142/S0219686714500085.

[29] Sivasankaran P. and Shahabudeen, P. M. (2016). Design and Comparison of Genetic Algorithms for Mixed-Model Assembly Line Balancing Problem with Original Task Times of Models, American Journal of Industrial and Business Management, vol. 6, p. 674,

[30] T. Starkweather, S.McDaniel, K. E.Mathias, L. D. Whitley, C. Whitley, (1991) A comparison of genetic sequencing operators, in: Proceedings of the 4th International Conference on Genetic Algorithms, (R. K. Belew, L. B. Booker, Eds.), Morgan Kaufmann, San Francisco CA,69-76.

[31] Varun Kumar S G, and Dr R. Panneerselvam (2017). A Study of Crossover Operators for Genetic Algorithms to Solve VRP and its Variants and New Sinusoidal Motion Crossover Operator. International Journal of Computational Intelligence Research. Vol 13, Number 7, pp. $1717-1733$

[32] Vijaymurthi C.N. and Panneerselvam, R. (2010). GA Based Heuristic for Total Covering Problem. Industrial Engineering Journal. II. 24-28.

[33] Xu, Y. (2012). A New Variable Neighbourhood Search Algorithm for the Multi-Depot Heterogeneous Vehicle Routing Problem with Time Windows, Electronic Notes in Discrete Mathematics. Vol- 39 pp.289-296. 